\title{
DIRAC OSCILLATOR IN DYNAMICAL NONCOMMUTATIVE SPACE
}

\author{
Ilyas Haouam* \\ Laboratoire de Physique Mathématique et de Physique Subatomique (LPMPS), \\ Université Frères Mentouri, Constantine 25000, Algeria
}

\begin{abstract}
In this paper, we address the energy eigenvalues of two-dimensional Dirac oscillator perturbed by dynamical noncommutative space. We derived the relativistic Hamiltonian of Dirac oscillator in dynamical noncommutative space $(\tau$-space), in which the space-space Heisenberg-like commutation relations and noncommutative parameter are position-dependent. Then used this Hamiltonian to calculate the first-order correction to the eigenvalues and eigenvectors, based on the second quantization and using the perturbation theory. It is shown that the energy shift depends on the dynamical noncommutative parameter $\tau$. Knowing that with a set of two-dimensional Bopp-shift transformation, we mapped the noncommutative problem to the standard commutative one.
\end{abstract}

Keywords: Dynamical noncommutative space, $\tau$-space, position-dependent noncommutativity, noncommutative space, Dirac oscillator.

\section{INTRODUCTION}

In the last few decades, physicists and mathematicians have developed a mathematical theory called noncommutative geometry, then quickly became a topic of great interest and has been finding applications in many areas of modern physics such as high energy [1], cosmology $[2,3]$, gravity [4], quantum physics [5-7] and field theory $[8,9]$. Substantially, the study on noncommutative (NC) spaces is very important for understanding phenomena at tiny scale of physical theories. Knowing that the idea behind extension of noncommutativity to the coordinates was first suggested by Heisenberg in 1930 as a solution to remove the infinite quantities of field theories. Besides, NC space-time structures are initiated by Snyder in 1947 $[10,11]$, in which he introduced noncommutativity in the hope of regularizing the divergencies that plagued quantum field theory.

Motivated by the attempts to understand string theory, the quantum gravitation and black holes through NC spaces and by seeking to highlight more phenomenological implications, we consider the Dirac oscillator (DO) within two-dimensional dynamical noncommutative (DNC) space.

Unlike the simplest possible type of NC spaces, in which $\mathrm{NC}$ parameter is constant, here we talk about a different type of $\mathrm{NC}$ spaces, where the deformation parameter will no longer be constant. However, there are many other possibilities that cannot be excluded. In fact, in the first paper by Snyder himself [10], the noncommutativity parameter was taken to depend on the coordinates and the momenta. Considerable different possibilities have been explored since then especially in the Lie-algebraic approaches [12], $\kappa$-Poincaré noncommutativity [13], other fuzzy spaces [14]. Besides, more recently

\footnotetext{
* ilyashaouam@live.fr ; ilyashaouam@ymail.com
}

in position-dependent approach [15-17], the authors considered $\Theta_{\mu \nu}$ to be a function of the position coordinates, i.e. $\Theta \rightarrow \Theta(X, Y)$.

The relativistic DO is very important potential for both theory and application. The potential term is introduced linearly by substitution $\vec{p} \rightarrow \vec{p}-i m \beta \omega \vec{r}$ in free Dirac Hamiltonian, this was considered for the first time by Ito et al [18], with $\vec{r}$ being the position vector and $m, \beta, \omega>0$ are the rest mass of the particle, Dirac matrix and constant oscillator frequency respectively. It is known as Dirac oscillator by Moshinsky and Szczepaniak [19] because it is a relativistic generalization of the nonrelativistic harmonic oscillator, exactly in non-relativistic limit it reduces to a standard harmonic oscillator with a strong spin-orbit coupling term.

Physically, DO has attracted a lot of attention because of its considerable physical applications, it is widely studied and illustrated. It can be shown that it is a physical system, which can be interpreted as the interaction of the anomalous magnetic moment with a linear electric field [20]. In addition, it can be associated with the electromagnetic potential [21]. As an exactly solvable model, DO in the background of a perpendicular uniform magnetic field have been wildly studied. However, we mention, for instance, the following: In ref. [19], the spectra of $(3+1)$-dimensional DO are solved and non-relativistic limit is discussed, as well, in ref [22], the symmetrical properties of the DO are studied. The operators of shift for symmetries are constructed explicitly [23]. Interestingly, the DO may afford a new approach to study quantum optics, where it was found that there is an exact map from (2+1)-dimensional DO to Jaynes-Cummings (JC) model [24], which describes the atomic transitions in a two level system. Subsequently, it found be that this model can be mapped either to JC or anti-JC models, depending on the magnitude of the magnetic field [25].

Basically, DO became more and more important since the experimental observations. For instance, we mention that Franco-Villafañe et al [26] exposed the proposal of 
the first-experimental microwave realization of the onedimensional DO. The experiment depends on a relation of the DO to a corresponding tight-binding system. The experimental results obtained, where the spectrum of the one-dimensional DO is in good agreement with that of the theory. Quimbay et al $[27,28]$ show that the DO may describe a naturally occurring physical system. Precisely, the case of a two-dimensional DO can be used to describe the dynamics of the charge carriers in graphene, and hence its electronic properties [29].

This paper is organized as follows. In section II, the DNC geometry is briefly reviewed. In section III, the two-dimensional DNC DO is investigated, where in subsection IIIB, the energy spectrum in noncommutative space is obtained. In sub-section III C, based on the perturbation theory and Fock basis, the energy spectrum including dynamical noncommutativity effect is obtained, therefore, we summarize the results and discussions. Section IV, is devoted to the conclusions.

\section{REVIEW OF POSITION-DEPENDENT NONCOMMUTATIVITY}

Let us present the essential formulas of the DNC space algebra we need in this study. As known at the tiny scale (string scale), the position coordinates do not commute with each other, thus the canonical variables satisfy the following deformed Heisenberg commutation relation

$$
\left[x_{\mu}^{n c}, x_{\nu}^{n c}\right]=i \Theta_{\mu \nu},
$$

with $\Theta_{\mu \nu}$ is an anti-symmetric tensor. The deformation parameter is a real constant and has the dimension of length ${ }^{2}$. Differently, here in the new version of NC spaces, $\Theta_{\mu \nu}$ is taken to be a function of coordinates. However, as a deformation of this NC parameter form will almost inevitably lead to non-Hermitian coordinates, it was pointed out recently [30] that these types of structures are related directly to non-Hermitian Hamiltonian systems. Thus, it is another subject to deal with later. Recently, Fring et al [16] made a generalization of $\mathrm{NC}$ space to a position-dependent space by introducing a set of new variables $X, Y, P_{x}, P_{y}$ and convert the constant $\Theta$ into a function $\Theta \rightarrow \Theta(X, Y)$, by choosing as one possibility $\theta(X, Y)=\Theta\left(1+\tau Y^{2}\right)$. In addition, Gomes $\mathrm{M}$ et all chose in their study [17] $\theta(X, Y)=\Theta /\left[1+\Theta \alpha\left(1+Y^{2}\right)\right]$.

It is interesting to note that $\sqrt{\Theta}$ has the dimension of length $(L)$, while $\sqrt{\tau}$ has the dimension of energy (or $L^{-1} \equiv m^{-1}$, see eq.(2)).

The new version of noncommutativity known as the DNC space or $\tau$-space. We restrict ourselves here to the two-dimensional space, the commutation relations (Lie brackets) are [16]

$$
\begin{array}{lccc}
{[X, Y]=} & i \Theta\left(1+\tau Y^{2}\right), & {\left[Y, P_{y}\right]=} & i \hbar\left(1+\tau Y^{2}\right), \\
{\left[X, P_{x}\right]=} & i \hbar\left(1+\tau Y^{2}\right), & {\left[Y, P_{x}\right]=} & 0, \\
{\left[X, P_{y}\right]=} & 2 i \tau Y\left(\Theta P_{y}+\hbar X\right),\left[P_{x}, P_{y}\right]= & 0 .
\end{array}
$$

In the limit $\tau \rightarrow 0$, it should be noted that we recover the non-DNC variables, therefore the $\mathrm{NC}$ variables satisfy the following Lie brackets

$$
\begin{aligned}
& {\left[x^{n c}, y^{n c}\right]=i \Theta,\left[y^{n c}, p_{y}^{n c}\right]=i \hbar} \\
& {\left[x^{n c}, p_{x}^{n c}\right]=i \hbar, \quad\left[y^{n c}, p_{x}^{n c}\right]=0} \\
& {\left[x^{n c}, p_{y}^{n c}\right]=0, \quad\left[p_{x}^{n c}, p_{y}^{n c}\right]=0 .}
\end{aligned}
$$

The coordinate $X$ and the momentum $P_{y}$ are not Hermitian, which make the Hamiltonian that includes these variables non-Hermitian. We may represent algebra (2) in terms of the standard Hermitian NC variables operators $x^{n c}, y^{n c}, p_{x}^{n c}, p_{y}^{n c}$ as

$$
\begin{array}{ll}
X=\left(1+\tau\left(y^{n c}\right)^{2}\right) x^{n c}, & Y=y^{n c}, \\
P_{y}=\left(1+\tau\left(y^{n c}\right)^{2}\right) p_{y}^{n c}, & P_{x}=p_{x}^{n c} .
\end{array}
$$

From this representation, we can see that some of the operators involved above are no longer Hermitian. However, to convert the non-Hermitian variables into a Hermitian one, we use a similarity transformation as a Dyson map $\eta O \eta^{-1}=o=O^{\dagger}$ (with $\left.\eta=\left(1+\tau Y^{2}\right)^{-\frac{1}{2}}\right)$, as stated in [16]. Therefore, we express the new Hermitian variables $x, y, p_{x}$ and $p_{y}$ in terms of $\mathrm{NC}$ variables as follows

$$
\begin{aligned}
x=\eta X \eta^{-1} & =\left(1+\tau Y^{2}\right)^{-\frac{1}{2}} X\left(1+\tau Y^{2}\right)^{\frac{1}{2}} \\
& =\left(1+\tau\left(y^{n c}\right)^{2}\right)^{\frac{1}{2}} x^{n c}\left(1+\tau\left(y^{n c}\right)^{2}\right)^{\frac{1}{2}} \\
y=\eta Y \eta^{-1} & =\left(1+\tau\left(y^{n c}\right)^{2}\right)^{-\frac{1}{2}} y^{n c}\left(1+\tau\left(y^{n c}\right)^{2}\right)^{\frac{1}{2}}=y^{n c} \\
p_{x}=\eta P_{x} \eta^{-1} & =\left(1+\tau\left(y^{n c}\right)^{2}\right)^{-\frac{1}{2}} p_{x}^{n c}\left(1+\tau\left(y^{n c}\right)^{2}\right)^{\frac{1}{2}}=p_{x}^{n c} \\
p_{y}=\eta P_{y} \eta^{-1} & =\left(1+\tau\left(y^{n c}\right)^{2}\right)^{-\frac{1}{2}} P_{y}\left(1+\tau\left(y^{n c}\right)^{2}\right)^{\frac{1}{2}} \\
& =\left(1+\tau\left(y^{n c}\right)^{2}\right)^{\frac{1}{2}} p_{y}^{n c}\left(1+\tau\left(y^{n c}\right)^{2}\right)^{\frac{1}{2}} .
\end{aligned}
$$

These new Hermitian DNC variables satisfy the following commutation relations

$$
\begin{array}{rrrrr}
{[x, y]} & =i \Theta\left(1+\tau y^{2}\right), & {\left[y, p_{y}\right]=} & i \hbar\left(1+\tau y^{2}\right) \\
{\left[x, p_{x}\right]=i \hbar\left(1+\tau y^{2}\right),} & {\left[y, p_{x}\right]=} & 0, \\
{\left[x, p_{y}\right]=2 i \tau y\left(\Theta p_{y}+\hbar x\right),} & {\left[p_{x}, p_{y}\right]=} & 0 .
\end{array}
$$

Now, using Bopp-shift transformation, one can express the $\mathrm{NC}$ variables in terms of the standard commutative variables [31]

$$
\begin{array}{ll}
x^{n c}=x^{s}-\frac{\Theta}{2 \hbar} p_{y}^{s}, & p_{x}^{n c}=p_{x}^{s}, \\
y^{n c}=y^{s}+\frac{\Theta}{2 \hbar} p_{y}^{s}, & p_{y}^{n c}=p_{y}^{s},
\end{array}
$$

where the index $s$ refers to the standard commutative space. The interesting point is that in the DNC space there is a minimum length for $X$ in a simultaneous $X, Y$ measurement [16]:

$$
\triangle X_{\min }=\Theta \sqrt{\tau} \sqrt{1+\tau\langle Y\rangle_{\rho}^{2}}
$$

as well, in a simultaneous $Y, P_{y}$ measurement we find a minimal momentum as

$$
\triangle\left(P_{y}\right)_{\min }=\hbar \sqrt{\tau} \sqrt{1+\tau\langle Y\rangle_{\rho}^{2}} .
$$




\section{TWO-DIMENSIONAL DIRAC OSCILLATOR IN DYNAMICAL NONCOMMUTATIVE SPACE}

\section{A. Extension to Dynamical Noncommutative Space}

The dynamics of the DO in the presence of a uniform external magnetic field is governed by the following Hamiltonian

$$
H_{D}=c \vec{\alpha} \cdot\left(\vec{p}^{s}-\frac{e}{c} \vec{A}^{s}-i m c \omega \beta \vec{r}^{s}\right)+\beta m c^{2}
$$

where $\vec{A}^{s}\left(A_{x}^{s}, A_{y}^{s}, A_{z}^{s}\right)$ is the vector potential produced by the external magnetic field, $e$ is the charge of the Dirac oscillator (the charge of the electron). The $\vec{\alpha}$ matrices, in two dimensions, are represented by the following Pauli matrices

$$
\begin{aligned}
& \alpha_{1}=\sigma_{x}=\left(\begin{array}{cc}
0 & 1 \\
1 & 0
\end{array}\right), \quad \alpha_{2}=\sigma_{y}=\left(\begin{array}{cc}
0 & -i \\
i & 0
\end{array}\right), \\
& \beta=\sigma_{z}=\left(\begin{array}{cc}
1 & 0 \\
0 & -1
\end{array}\right),
\end{aligned}
$$

which, satisfy the commutation relation

$$
\begin{gathered}
\alpha_{i}^{2}=\beta^{2}=1 \\
\alpha_{i} \alpha_{j}+\alpha_{j} \alpha_{i}=0, i=1,2,3 . \\
\alpha_{i} \beta+\beta \alpha_{i}=0
\end{gathered}
$$

In two dimensions, equation (10) becomes

$$
\begin{aligned}
H_{D}=c & \left(\alpha_{1} p_{x}^{s}+\alpha_{2} p_{y}^{s}\right)-e\left(\alpha_{1} A_{x}^{s}+\alpha_{2} A_{y}^{s}\right) \\
& -i m c \omega\left(\alpha_{1} \beta x^{s}+\alpha_{2} \beta y^{s}\right)+\beta m c^{2}
\end{aligned}
$$

Let us choose the direction of the field $\vec{B}$ according to $(\mathrm{Oz})$, then the vector potential $\vec{A}^{s}$ is given, in the Landau gauge, by

$$
\vec{A}=\frac{B}{2}\left(-y^{s}, x^{s}, 0\right)
$$

therefore, we have

$$
\begin{aligned}
H_{D}\left(x_{i}^{s}, p_{i}^{s}\right)=c & \left(\alpha_{1} p_{x}^{s}+\alpha_{2} p_{y}^{s}\right)+e \frac{B}{2}\left(\alpha_{1} y^{s}-\alpha_{2} x^{s}\right) \\
& -i m c \omega\left(\alpha_{1} \beta x^{s}+\alpha_{2} \beta y^{s}\right)+\beta m c^{2}
\end{aligned}
$$

The Hamiltonian of a two-dimensional DO in DN space is given by

$$
\begin{aligned}
H_{D}\left(x_{i}, p_{i}\right)= & c\left(\alpha_{1} p_{x}+\alpha_{2} p_{y}\right)+e \frac{B}{2}\left(\alpha_{1} y+\alpha_{2} x\right) \\
& -i m c \omega\left(\alpha_{1} \beta x+\alpha_{2} \beta y\right)+\beta m c^{2} .
\end{aligned}
$$

Now, using equation (5), we express the Hamiltonian above in terms of $\mathrm{NC}$ variables

$$
\begin{gathered}
H_{D}\left(x_{i}^{n c}, p_{i}^{n c}\right)=\beta m c^{2} \\
+c\left[\alpha_{1} p_{x}^{n c}+\alpha_{2}\left(1+\tau\left(y^{n c}\right)^{2}\right)^{\frac{1}{2}} p_{y}^{n c}\left(1+\tau\left(y^{n c}\right)^{2}\right)^{\frac{1}{2}}\right] \\
+e \frac{B}{2}\left[\alpha_{1} y^{n c}-\alpha_{2}\left(1+\tau\left(y^{n c}\right)^{2}\right)^{\frac{1}{2}} x^{n c}\left(1+\tau\left(y^{n c}\right)^{2}\right)^{\frac{1}{2}}\right] \\
-i m c \omega\left[\alpha_{1} \beta\left(1+\tau\left(y^{n c}\right)^{2}\right)^{\frac{1}{2}} x^{n c}\left(1+\tau\left(y^{n c}\right)^{2}\right)^{\frac{1}{2}}+\alpha_{2} \beta y^{n c}\right] .
\end{gathered}
$$

Since $\tau$ is very small, the parentheses can be expanded to the first order using

$$
\left(1+\tau\left(y^{n c}\right)^{2}\right)^{\frac{1}{2}}=1+\frac{1}{2} \tau\left(y^{n c}\right)^{2},
$$

so that, equation (17) turns to

$$
\begin{gathered}
H_{D}\left(x_{i}^{n c}, p_{i}^{n c}\right)=c\left[\alpha_{1} p_{x}^{n c}+\alpha_{2}\left\{p_{y}^{n c}+\frac{1}{2} \tau\left(y^{n c}\right)^{2} p_{y}^{n c}+\frac{1}{2} \tau p_{y}^{n c}\left(y^{n c}\right)^{2}\right\}\right] \\
+\beta m c^{2}+e \frac{B}{2}\left[\alpha_{1} y^{n c}-\alpha_{2}\left\{x^{n c}+\frac{1}{2} \tau\left(y^{n c}\right)^{2} x^{n c}+\frac{1}{2} \tau x^{n c}\left(y^{n c}\right)^{2}\right\}\right] \\
\quad-i m c \omega\left[\alpha_{2} \beta y^{n c}+\alpha_{1} \beta\left\{x^{n c}+\frac{1}{2} \tau\left(y^{n c}\right)^{2} x^{n c}+\frac{1}{2} \tau x^{n c}\left(y^{n c}\right)^{2}\right\}\right] .
\end{gathered}
$$

Using the Bopp-shift transformation (7) now, Hamiltonian (19) can be expressed in terms of the standard commutative variables

$$
\begin{gathered}
H_{D}\left(x_{i}^{s}, p_{i}^{s}\right)=c \alpha_{1} p_{x}^{s}+\beta m c^{2}+c \alpha_{2} p_{y}^{s} \\
+c \alpha_{2}\left\{\frac{1}{2} \tau\left(y^{s}+\frac{\Theta}{2 \hbar} p_{x}^{s}\right)^{2} p_{y}^{s}+\frac{1}{2} \tau p_{y}^{s}\left(y^{s}+\frac{\Theta}{2 \hbar} p_{x}^{s}\right)^{2}\right\} \\
+\frac{e B}{2}\left[\alpha_{1}\left(y^{s}+\frac{\Theta}{2 \hbar} p_{x}^{s}\right)-\alpha_{2}\left\{\frac{\tau}{2}\left(y^{s}+\frac{\Theta}{2 \hbar} p_{x}^{s}\right)^{2}\left(x^{s}-\frac{\Theta}{2 \hbar} p_{y}^{s}\right)\right.\right. \\
\left.\left.x^{s}-\frac{\Theta}{2 \hbar} p_{y}^{s}+\frac{1}{2} \tau\left(x^{s}-\frac{\Theta}{2 \hbar} p_{y}^{s}\right)\left(y^{s}+\frac{\Theta}{2 \hbar} p_{x}^{s}\right)^{2}\right\}\right] \\
-i m c \omega\left[\alpha_{2} \beta\left(y^{s}+\frac{\Theta}{2 \hbar} p_{x}^{s}\right)+\alpha_{1} \beta\left\{x^{s}-\frac{\Theta}{2 \hbar} p_{y}^{s}\right.\right. \\
\left.\left.+\frac{\tau}{2}\left(y^{s}+\frac{\Theta}{2 \hbar} p_{x}^{s}\right)^{2}\left(x^{s}-\frac{\Theta}{2 \hbar} p_{y}^{s}\right)+\frac{\tau}{2}\left(x^{s}-\frac{\Theta}{2 \hbar} p_{y}^{s}\right)\left(y^{s}+\frac{\Theta}{2 \hbar} p_{x}^{s}\right)^{2}\right\}\right] .
\end{gathered}
$$

Therefore, to the first order in $\Theta$ and $\tau$, we have (noting that terms containing $\Theta \tau$ are also neglected)

$$
\begin{aligned}
& H_{D}\left(x_{i}^{s}, p_{i}^{s}\right)=c\left[\alpha_{1} p_{x}^{s}+\alpha_{2}\left\{p_{y}^{s}+\frac{\tau}{2}\left(y^{s}\right)^{2} p_{y}^{s}+\frac{1}{2} \tau p_{y}^{s}\left(y^{s}\right)^{2}\right\}\right] \\
& +\beta m c^{2}+e \frac{B}{2}\left[\alpha_{1}\left(y^{s}+\frac{\Theta}{2 \hbar} p_{x}^{s}\right)-\alpha_{2}\left\{x^{s}-\frac{\Theta}{2 \hbar} p_{y}^{s}+\tau x^{s}\left(y^{s}\right)^{2}\right\}\right] \\
& -i m c \omega\left[\alpha_{2} \beta\left(y^{s}+\frac{\Theta}{2 \hbar} p_{x}^{s}\right)+\alpha_{1} \beta\left\{x^{s}-\frac{\Theta}{2 \hbar} p_{y}^{s}+\tau x^{s}\left(y^{s}\right)^{2}\right\}\right],
\end{aligned}
$$

which can be written as

$$
H_{D}=H_{\Theta}+H_{\tau},
$$

with

$$
\begin{gathered}
H_{\Theta}=c \alpha_{1} p_{x}^{s}+c \alpha_{2} p_{y}^{s}+\frac{e B}{2}\left(\alpha_{1} y^{s}-\alpha_{2} x^{s}\right) \\
-i m c \omega\left(\alpha_{1} \beta x^{s}+\alpha_{2} \beta y^{s}\right)+\beta m c^{2} \\
+\frac{\Theta}{2 \hbar}\left\{\frac{e B}{2}\left(\alpha_{1} p_{x}^{s}+\alpha_{2} p_{y}^{s}\right)-i m c \omega\left(\alpha_{2} \beta p_{x}^{s}-\alpha_{1} \beta p_{y}^{s}\right)\right\} \\
H_{\tau}=\frac{1}{2} \tau\left\{c \alpha_{2}\left(y^{s}\right)^{2} p_{y}^{s}+c \alpha_{2} p_{y}^{s}\left(y^{s}\right)^{2}\right. \\
=\frac{\tau}{2}\left\{\alpha_{2} \mathcal{V}_{1}+\alpha_{2} \mathcal{V}_{2}-\left(e B \alpha_{2}+i 2 m c \omega \alpha_{1} \beta\right) \mathcal{V}_{3}\right\}
\end{gathered}
$$

Knowing that $H_{\tau}$ is the perturbation Hamiltonian in which it reflects the effects of dynamical noncommutativity of space on the deformed Dirac Hamiltonian. We also can treat the term proportional to $\Theta$ in equation (23) as a perturbation term. While the noncommutativity parameter $\tau$ is non-zero and very small, we use the perturbation theory to find the spectrum of the systems in question.

The two-dimensional DO equation in DNC space is written as follow

$$
H_{D}\left|\psi_{D}\right\rangle=\left(H_{\Theta}+H_{\tau}\right)\left|\psi_{D}\right\rangle=E_{\Theta, \tau}\left|\psi_{D}\right\rangle
$$


with

$$
\left|\psi_{D}\right\rangle=\left(\left|\psi_{1}\right\rangle,\left|\psi_{2}\right\rangle\right)^{T}
$$

is the wave function of the system in question.

\section{B. Unperturbed Eigenvalues and Eigenvectors}

We introduce the following complex coordinates

$$
\begin{aligned}
& z^{s}=x^{s}+i y^{s}, \bar{z}^{s}=x^{s}-i y^{s}, \\
& p_{z}^{s}=-i \hbar \frac{d}{d z^{s}}=\frac{1}{2}\left(p_{x}^{s}-i p_{y}^{s}\right), \\
& p_{\bar{z}}^{s}=-i \hbar \frac{d}{d \bar{z}^{s}}=\frac{1}{2}\left(p_{x}^{s}+i p_{y}^{s}\right)
\end{aligned}
$$

where

$$
\left[z^{s}, p_{z}^{s}\right]=\left[\bar{z}^{s}, p_{\bar{z}}^{s}\right]=i \hbar,\left[z^{s}, p_{\bar{z}}^{s}\right]=\left[\bar{z}^{s}, p_{z}^{s}\right]=0 .
$$

Using equation (11), our unperturbed NC DO Hamiltonian (23) in the complex formalism simply becomes

$H_{\Theta}=\left[\begin{array}{cc}m c^{2} & \left(1+m \frac{\Theta \tilde{\omega}}{2 \hbar}\right) 2 c p_{z}^{s}+i m c \tilde{\omega} \bar{z}^{s} \\ \left(1+m \frac{\Theta \tilde{\omega}}{2 \hbar}\right) 2 p_{\bar{z}}^{s}-i m c \tilde{\omega} z^{s} & -m c^{s}\end{array}\right]$,

with

$$
\tilde{\omega}=\omega-\frac{\omega_{c}}{2}
$$

where $\omega_{c}=\frac{|e| B}{m c}$ is the cyclotron frequency. By putting $\Omega=1+m \frac{\Theta \tilde{\omega}}{2 \hbar}$ then our Hamiltonian defined by equation (30), becomes

$$
H_{\Theta}=\left[\begin{array}{cc}
m c^{2} & 2 c \Omega p_{z}^{s}+i m c \bar{z}^{s} \tilde{\omega} \\
2 c \Omega p_{\bar{z}}^{s}-i m c \omega z^{s} \tilde{\omega} & -m c^{2}
\end{array}\right] .
$$

Now, we move to the formalism of the second quantization. Let us introduce the following creation and annihilation operators

$$
\begin{aligned}
a & =i\left(\frac{\Omega}{\sqrt{m \bar{\omega} \hbar}} p_{\bar{z}}^{s}-\frac{i}{2 \Omega} \sqrt{\frac{m \bar{\omega}}{\hbar}} z^{s}\right), \\
a^{\dagger} & =-i\left(\frac{\Omega}{\sqrt{m \bar{\omega} \hbar}} p_{z}^{s}+\frac{i}{2 \Omega} \sqrt{\frac{m \bar{\omega}}{\hbar}} \bar{z}^{s}\right) .
\end{aligned}
$$

The operators above satisfy the following commutations relations

$$
\left[a, a^{\dagger}\right]=1,[a, a]=\left[a^{\dagger}, a^{\dagger}\right]=0
$$

Thus, our Hamiltonian (32) is written in terms of the creation and annihilation operators by

$$
H_{\Theta}=\left[\begin{array}{cc}
m c^{2} & i 2 c \sqrt{m \bar{\omega} \hbar} a^{\dagger} \\
-i 2 c \sqrt{m \bar{\omega} \hbar} a & -m c^{2}
\end{array}\right]=\left[\begin{array}{cc}
m c^{2} & i g a^{\dagger} \\
-i g a & -m c^{2}
\end{array}\right],
$$

with the parameter $\bar{g}=2 c \sqrt{m \hbar \bar{\omega}}$ describes the coupling between different states in NC space, and $\bar{\omega}=\Omega \tilde{\omega}$ is the correction of the frequency $\tilde{\omega}$ of commutative space. Besides, parameter $g=2 c \sqrt{m \tilde{\omega} \hbar}$ describes the coupling between different states in commutative space. Now, we try to solve the following equation

$$
H_{\Theta}\left|\bar{\psi}_{D}\right\rangle=E_{\Theta}\left|\bar{\psi}_{D}\right\rangle
$$

where $E_{\Theta},\left|\bar{\psi}_{D}\right\rangle$ are the eigenenergy and wave function of the Dirac equation above. By placing equation (26) in (37), we obtain the following system of equations

$$
\left(\begin{array}{cc}
m c^{2} & i \bar{g} a^{\dagger} \\
-i \bar{g} a & -m c^{2}
\end{array}\right)\left(\begin{array}{l}
\mid \bar{\psi}_{1}> \\
\mid \bar{\psi}_{2}>
\end{array}\right)=E_{\Theta}\left(\begin{array}{l}
\mid \bar{\psi}_{1}> \\
\mid \bar{\psi}_{2}>
\end{array}\right)
$$

where

$$
\begin{gathered}
\left(m c^{2}-E_{\Theta}\right)\left|\bar{\psi}_{1}>+i \bar{g} a^{\dagger}\right| \bar{\psi}_{2}>=0, \\
-i \bar{g} a\left|\bar{\psi}_{1}>-\left(m c^{2}+E_{\Theta}\right)\right| \bar{\psi}_{2}>=0 .
\end{gathered}
$$

From the equations (39) and (40), we have

$$
\left|\bar{\psi}_{2}>=\frac{-i \bar{g} a^{\dagger}}{E_{\Theta}+m c^{2}}\right| \bar{\psi}_{1}>
$$

subsequently

$$
\left[\bar{g}^{2} a^{\dagger} a+m^{2} c^{4}-\left(E_{\Theta}\right)^{2}\right] \mid \bar{\psi}_{1}>=0 .
$$

In the basis of the second quantization, of which $\left|\bar{\psi}_{1}>\equiv\right| n>$, we have

$$
\left[\bar{g}^{2} n+m^{2} c^{4}-\left(E_{\Theta}\right)^{2}\right]\left|n>=0, a^{\dagger} a\right| n>=n \mid n>.
$$

Thus, the energy spectrum is given by

$$
E_{\Theta, n}^{ \pm}= \pm \sqrt{m^{2} c^{4}+\bar{g}^{2} n}
$$

which can be rewritten as

$$
E_{\Theta, n}^{ \pm}= \pm m c^{2} \sqrt{1+\frac{4 \hbar \tilde{\omega}}{m c^{2}}\left(1+m \frac{\Theta \tilde{\omega}}{2 \hbar}\right)} n, n=0,1,2, \ldots
$$

Furthermore, we have the reduced energy spectrum

$$
\frac{E_{n}^{ \pm}}{E_{0}}= \pm \sqrt{1+4 w\left(1+\frac{1}{2} q w\right) n}
$$

where $w=\frac{\hbar \tilde{\omega}}{m c^{2}}$, is a parameter that controls the nonrelativistic limit, also $E_{0}=m c^{2}$ is a background energy, which corresponds to $n=0$. And $q=\frac{\Theta}{\Theta_{0}}$ with $\Theta_{0}=$ $\left(\frac{\hbar}{m c}\right)^{2}$ of the dimension $\left[\frac{\hbar}{m c}\right]^{2}=L^{2} \equiv m^{2}$.

The corresponding wave function is written as a function of the basis $\left|n>=\frac{\left(a^{\dagger}\right)^{n}}{\sqrt{n !}}\right| 0>$, and it is given by the following formula

$$
\left|\bar{\psi}_{n}^{ \pm}>=c_{n}^{ \pm}\right| n ; \frac{1}{2}>+d_{n}^{ \pm} \mid n-1 ;-\frac{1}{2}>,
$$


where the coefficients $c_{n}^{ \pm}$and $d_{n}^{ \pm}$are determined from the normalization condition. We thus obtain

$$
c_{n}^{ \pm}= \pm \sqrt{\frac{E_{n}^{+} \pm m c^{2}}{2 E_{n}^{+}}}, d_{n}^{ \pm}=\mp \sqrt{\frac{E_{n}^{+} \mp m c^{2}}{2 E_{n}^{+}}} .
$$

In the limit $\Theta \rightarrow 0$, the $\mathrm{NC}$ energy spectrum becomes commutative one, i.e. equation (45) turns into equation (10) of ref [32], which confirms that we are in good agreement. As well, in ref [33] Boumali et al made a study of DO in NC phase-space, where if $\bar{\Theta} \rightarrow 0$, the energy eigenvalues (eq. 50) will be similar as of ours in equation (45).

We plot the reduced energy spectrum in terms of quantum number $n$, for the cases $w=1, q=1 ; q=1, q=2$ and commutative case with $w=1$.

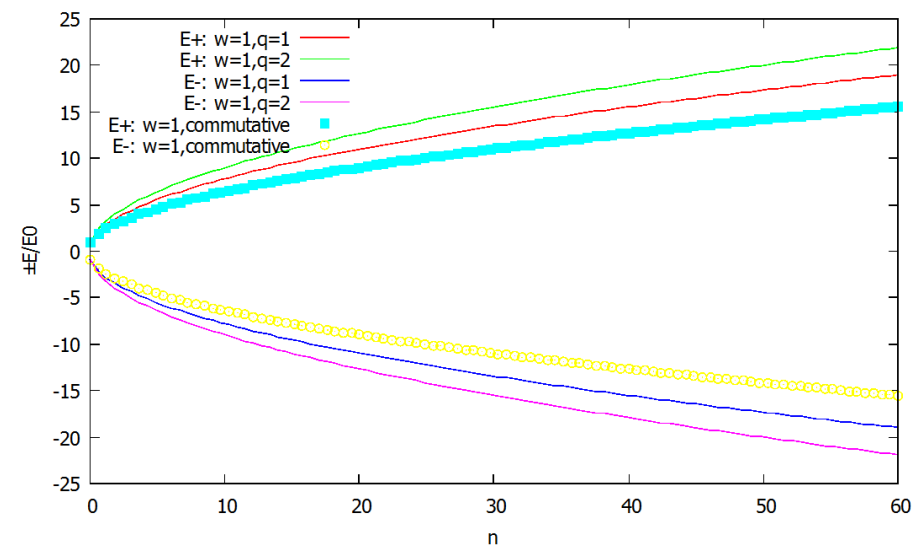

Figure 1. A reduced energy versus quantum number in both cases of $\mathrm{NC}$ and commutative spaces.

The $\frac{E_{n}^{ \pm}}{E_{0}}$ as a function of the quantum number $\mathrm{n}$ of equation (46) in both commutative $(\Theta=q=0)$ and $\mathrm{NC}$ $(q=1 ; q=2)$ spaces are illustrated in fig. 1 . Knowing that fig. 1 discloses that the influence of the $\mathrm{NC}$ parameter on the energy spectrum is considerable and significant.

The following figure shows the coupling parameters $g$ and $\bar{g}$, between different levels for the two cases in NC space. (a)

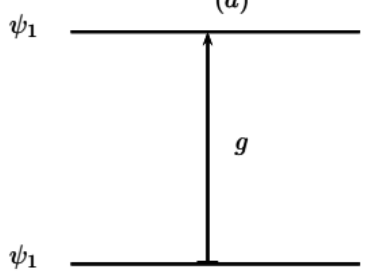

(b)

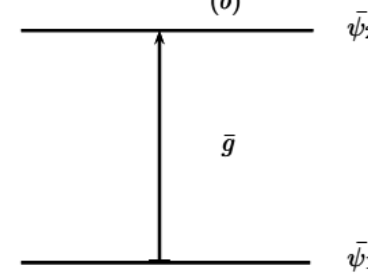

Figure 2. The coupling parameter between different levels : (a) Case of commutative space, (b) Case of NC space
While $n$ are non-negative integers, we observe that these eigenvalues are non-degenerated. Consequently, we can see that our spectrum has no degeneracy. But differently, for instance, energy levels can appear degenerated, as in a study [34] about the mesoscopic states in a relativistic Landau levels, the authors found that the energy spectrum is dependent on $p_{z}^{2}$ (check eq. 13 in this cited reference), which is the underlying reasons for infinite degeneracy of all levels.

\section{Perturbed System}

We aim in this sub-section to determine the correction of first-order energy by using first-order energy shift formulas. To explain the structure of our spectrum, we will use time-independent perturbation theory for small values of the $\mathrm{NC}$ parameter $\tau$. In view that energies are non-degenerated, we use the non-degenerated timeindependent perturbation theory

$$
\begin{gathered}
\left|\overline{\psi_{n}}\right\rangle=\left|\bar{\psi}_{n}^{(0)}\right\rangle+\tau\left|\bar{\psi}_{n}^{(1)}\right\rangle+\tau^{2}\left|\bar{\psi}_{n}^{(2)}\right\rangle+\ldots \\
E_{n}=E_{n}^{(0)}+\tau E_{n}^{(1)}+\tau^{2} E_{n}^{(2)}+\ldots
\end{gathered}
$$

Here the (0) superscript denote the quantities that are associated with the unperturbed system.

The first-order correction to the eigenvalues and eigenvectors in perturbation theory are simply given by

$$
\begin{array}{r}
E_{n}^{(1)}=\triangle E_{n}=<\bar{\psi}_{n}^{(0)}\left|\frac{1}{\tau} H_{\tau}\right| \bar{\psi}_{n}^{(0)}>, \\
\left|\bar{\psi}_{n}^{(1)}\right\rangle=\sum_{k \neq n} \frac{<\bar{\psi}_{k}^{(0)}\left|\frac{1}{\tau} H_{\tau}\right| \bar{\psi}_{n}^{(0)}>}{E_{n}^{(0)}-E_{m}^{(0)}}\left|\bar{\psi}_{k}^{(0)}\right\rangle .
\end{array}
$$

Inserting equation (24) into equation above, we find $E_{n}^{(1)}=<\bar{\psi}_{n}^{(0)}\left|i\left\{\alpha_{2}\left(\mathcal{V}_{1}+\mathcal{V}_{2}\right)-\left(e B \alpha_{2}+i 2 m c \omega \alpha_{1} \beta\right) \mathcal{V}_{3}\right\}\right| \bar{\psi}_{n}^{(0)}>$,

the operator method can also be used to obtain the energy shift in Fock space. In our scenario, we require adopting the notation of the state as follows

$$
\left|\bar{\psi}_{n}^{(0)}>=\right| n_{x}, n_{y}>\text {. }
$$

The perturbation matrix is given by

$\left.\mathcal{M}=<n_{x}, n_{y}\left|i\left(\begin{array}{cc}0 & -\left(\mathcal{V}_{1}+\mathcal{V}_{2}\right)+\Upsilon \mathcal{V}_{3} \\ \mathcal{V}_{1}+\mathcal{V}_{2}-\Upsilon \mathcal{V}_{3} & 0\end{array}\right)\right| n_{x}^{\prime}, n_{y}^{\prime}\right\rangle$,

with $\Upsilon=e B+2 m c \omega$. To calculate the action of $\mathcal{V}_{i}(i=$ $1, . ., 3)$ on the element of the Fock basis, we conveniently use the following $b_{j}, b_{j}^{\dagger}(j=x, y)$ operators

$b_{j}=\sqrt{\frac{m \omega}{2 \hbar}}\left(x_{j}+i \frac{p_{j}}{m \omega}\right)$ and $b_{j}^{\dagger}=\sqrt{\frac{m \omega}{2 \hbar}}\left(x_{j}-i \frac{p_{j}}{m \omega}\right)$, 
where

$$
\left[b_{j}^{\dagger}, b_{j}\right]=1, \text { with } b_{j}^{\dagger} b_{j}=n_{j} .
$$

With the help of the following definitions of eigenkets and central properties of creation and annihilation operators [35]

$$
\begin{aligned}
b_{j} \mid n_{j}> & =\sqrt{n_{j}} \mid n_{j}-1>, \\
b_{j}^{\dagger} \mid n_{j}> & =\sqrt{n_{j}+1} \mid n_{j}+1>, \\
b_{j}^{2} \mid n_{j}> & =\sqrt{n_{j}\left(n_{j}-1\right)} \mid n_{j}-2>, \\
b_{j}^{\dagger 2} \mid n_{j}> & =\sqrt{\left(n_{j}+1\right)\left(n_{j}+2\right)} \mid n_{j}+2>,
\end{aligned}
$$

where $<n_{j}^{\prime} \mid n_{j}>=\delta_{n_{j}^{\prime}, n_{j}}$ and

$$
\left[b^{\dagger} b, b^{\dagger}\right]=b^{\dagger} b b^{\dagger}-b^{\dagger 2} b=b^{\dagger},\left[b^{\dagger} b, b\right]=b^{\dagger} b^{2}-b b^{\dagger} b=-b .
$$

The contributions of the different parts of the perturbed Hamiltonian are as follows

$$
\begin{aligned}
&<n_{x}, n_{y} \mid \mathcal{V}_{1}\left|n_{x}^{\prime}, n_{y}^{\prime}>=<n_{x}, n_{y}\right| \frac{c}{2}\left(y^{s}\right)^{2} p_{y}^{s} \mid n_{x}^{\prime}, n_{y}^{\prime}> \\
&=\frac{c \hbar}{4} \sqrt{\frac{\hbar}{2 m \omega}}\left[n_{y}^{\prime} \sqrt{n_{y}^{\prime}+1} \delta_{n_{X}, n_{X}^{\prime}} \delta_{n_{y}, n_{y}^{\prime}+1}\right. \\
&-\left(1+2 n_{y}^{\prime}-\sqrt{\left.n_{y}^{\prime 2}-1\right) \sqrt{n_{y}^{\prime}} \delta_{n_{X}, n_{X}^{\prime}} \delta_{n_{y}, n_{y}^{\prime}-1}}\right. \\
&-\sqrt{n_{y}^{\prime}\left(n_{y}^{\prime}-1\right)\left(n_{y}^{\prime}-2\right)} \delta_{n_{X}, n_{X}^{\prime}} \delta_{n_{y}, n_{y}^{\prime}-3} \\
&\left.+\sqrt{\left(n_{y}^{\prime}+1\right)\left(n_{y}^{\prime}+2\right)\left(n_{y}^{\prime}+3\right)} \delta_{n_{X}, n_{X}^{\prime}} \delta_{n_{y}, n_{y}^{\prime}+3}\right],
\end{aligned}
$$

$$
\begin{gathered}
<n_{x}, n_{y}\left|\mathcal{V}_{2}\right| n_{x}^{\prime}, n_{y}^{\prime}>=<n_{x}, n_{y}\left|\frac{c}{2} p_{y}^{s}\left(y^{s}\right)^{2}\right| n_{x}^{\prime}, n_{y}^{\prime}> \\
=\frac{c \hbar^{2}}{8 m \omega} \sqrt{\frac{\hbar}{2 m \omega}}\left[-\sqrt{n_{y}^{\prime}\left(n_{y}^{\prime}-1\right)\left(n_{y}^{\prime}-2\right)} \delta_{n_{X}, n_{X}^{\prime}} \delta_{n_{y}, n_{y}^{\prime}-3}\right. \\
+\sqrt{\left(n_{y}^{\prime}+1\right)\left(n_{y}^{\prime}+2\right)\left(n_{y}^{\prime}+3\right)} \delta_{n_{X}, n_{X}^{\prime}} \delta_{n_{y}, n_{y}^{\prime}+3} \\
\quad-\left(1+\sqrt{\left.n_{y}^{\prime 2}-1\right)} \sqrt{n_{y}^{\prime}} \delta_{n_{X}, n_{X}^{\prime}} \delta_{n_{y}, n_{y-1}^{\prime}}\right. \\
\left.+\left(2-n_{y}^{\prime}+\sqrt{n_{y}^{\prime 2}+2 n_{y}^{\prime}}\right) \sqrt{n_{y}^{\prime}+1} \delta_{n_{X}, n_{X}^{\prime}} \delta_{n_{y}, n_{y+1}^{\prime}}\right],
\end{gathered}
$$

$$
\begin{gathered}
\left.<n_{x}, n_{y}\left|\mathcal{V}_{3}\right| n_{x}^{\prime}, n_{y}^{\prime}\right\rangle=<n_{x}, n_{y}\left|\frac{1}{2} x^{s}\left(y^{s}\right)^{2}\right| n_{x}^{\prime}, n_{y}^{\prime}> \\
=\frac{1}{2} \sqrt{\frac{\hbar}{2 m \omega}}\left[\sqrt{n_{x}^{\prime}} \delta_{n_{x}, n_{x}^{\prime}-1}+\sqrt{n_{x}^{\prime}+1} \delta_{n_{x}, n_{x}^{\prime}+1}\right] \\
\times\left[\sqrt{n_{y}^{\prime}\left(n_{y}^{\prime}-1\right)} \delta_{n_{y}, n_{y}^{\prime}-2}\right. \\
\left.+\sqrt{\left(n_{y}^{\prime}+1\right)\left(n_{y}^{\prime}+2\right)} \delta_{n_{y}, n_{y}^{\prime}+2}+\left(2 n_{y}^{\prime}-1\right) \delta_{n_{y}, n_{y}^{\prime}}\right] .
\end{gathered}
$$

The relevant perturbation matrix is given by

$$
\mathcal{M}=i\left(\begin{array}{cc}
0 & \mathcal{W}_{12} \\
\mathcal{W}_{21} & 0
\end{array}\right)
$$

with

$$
\mathcal{W}_{12}=-\mathcal{W}_{21}=<n_{x}, n_{y}\left|\left\{\Upsilon \mathcal{V}_{3}-\left(\mathcal{V}_{1}+\mathcal{V}_{2}\right)\right\}\right| n_{x}^{\prime}, n_{y}^{\prime}>\text {. }
$$

The DO Hamiltonian $H_{D}=H_{\Theta}+H_{\tau}$ may be represented by a square matrix as follows (we have used the basis given by unperturbed energy eigenkets)

$$
H_{D} \equiv\left(\begin{array}{cc}
E_{n,+}^{(0)}(\Theta) & i \tau \mathcal{W}_{12} \\
i \tau \mathcal{W}_{21} & E_{n,-}^{(0)}(\Theta)
\end{array}\right)
$$

with $\mathcal{W}$ matrix must be Hermitian, the eigenvalued of the problem above are

$$
\left(\begin{array}{c}
E_{1} \\
E_{2}
\end{array}\right)=\frac{E_{+}^{(0)}+E_{-}^{(0)}}{2} \pm \sqrt{\frac{\left(E_{+}^{(0)}-E_{-}^{(0)}\right)^{2}}{4}+\lambda^{2}\left|\mathcal{W}_{12}\right|^{2}}
$$

Here we set $\lambda=i \tau$. Supposing that $\lambda\left|\mathcal{W}_{12}\right|$ is small compared to relevant energy scale, so that the difference of the energy eigenvalues of the umperturbed system

$$
\lambda\left|\mathcal{W}_{12}\right|<\left|E_{+}^{(0)}-E_{-}^{(0)}\right| .
$$

To obtain the expansion of the energy eigenvalues in the presence of perturbation, namely (a perturbation expansion always exists for a sufficiently weak perturbation)

$$
\begin{aligned}
& E_{1}=E_{-}^{(0)}+\frac{\lambda^{2}\left|\mathcal{W}_{12}\right|^{2}}{E_{-}^{(0)}-E_{+}^{(0)}}+\ldots \\
& E_{2}=E_{+}^{(0)}+\frac{\lambda^{2}\left|\mathcal{W}_{12}\right|^{2}}{E_{+}^{(0)}-E_{-}^{(0)}}+\ldots
\end{aligned}
$$

We terminate the calculation by the radius of convergence of series expansion (68), so while $\lambda$ is a complex variable, $\lambda$ is increased from zero, branch points are encoutered at [34]

$$
\lambda\left|\mathcal{W}_{12}\right|=\frac{ \pm i\left(E_{-}^{(0)}-E_{+}^{(0)}\right)}{2},
$$

the condtion for the convergence of (68) while the $\lambda=1$ full strength case is

$$
\left|\mathcal{W}_{12}\right|=\frac{\left|E_{-}^{(0)}-E_{+}^{(0)}\right|}{2}
$$

If this condition is not met, expansion (68) is meaningless.

It can be checked that all the results of the $\mathrm{NC}$ case can be obtained from the DNC case directly by taking the limit of $\tau \rightarrow 0$, for instance equations (49) and (50) give the same values as the eigenvalues and eigenvectors in $\mathrm{NC}$ space, i.e. equations (45) and (47), respectively.

It may be also useful to mention that the $\mathrm{NC}$ DO (in non-DNC space) has been investigated in [36-38].

We can regard equation (68) as the eigenvalues of our system, where we restrict ourselves to the first-order correction to the eigenvalues and eigenvectors, which leads the energy shift for the ground state. Besides, it is easy to obtain the eigensolutions for excited states.

It is interesting to illustrate the DNC effect on DO energy levels. This effect is reduced in the energy shifts obtained, hence we do the following sample, with

$$
\begin{aligned}
& a_{1}=\quad \frac{\Upsilon}{2} \sqrt{\frac{\hbar}{2 m \omega}}, \\
& a_{2}=\left(\frac{3 c \hbar}{4}+\frac{c \hbar^{2}}{8 m \omega}\right) \sqrt{\frac{\hbar}{2 m \omega}}, \\
& a_{3}=\quad \frac{c \hbar^{2}}{4 m \omega} \sqrt{\frac{\hbar}{2 m \omega}}, \\
& a_{4}=\left(\frac{c \hbar}{4}+\frac{2,73 c \hbar^{2}}{8 m \omega}\right) \sqrt{\frac{\hbar}{m \omega}} .
\end{aligned}
$$




\begin{tabular}{ccccc}
\hline$\left(n_{x}, n_{y}, n_{x}^{\prime}, n_{y}^{\prime}\right)$ & $\mathcal{W}_{12}$ & $E_{1}$ & $E_{2}$ & $\triangle E$ \\
\hline$(1,1,1,1)$ & 0 & $-5,11.10^{5}$ & $5,11.10^{5}$ & 0 \\
\hline$(1,0,0,0)$ & $-a_{1}$ & $-5,11.10^{5}+\frac{a_{1} \tau^{2}}{10,22.10^{5}}$ & $5,11.10^{5}-\frac{a_{1} \tau^{2}}{10,22.10^{5}}$ & $\frac{a_{1} \tau^{2}}{10,22.10^{5}}$ \\
\hline$(0,0,0,1)$ & $a_{2}$ & $-5,11.10^{5}+\frac{a_{2} \tau^{2}}{10,22.10^{5}}$ & $5,11.10^{5}-\frac{a_{2} \tau^{2}}{10,22.10^{5}}$ & $\frac{a_{2} \tau^{2}}{10,22.10^{5}}$ \\
\hline$(0,1,0,0)$ & $-a_{3}$ & $-5,11.10^{5}+\frac{a_{3} \tau^{2}}{10,22.10^{5}}$ & $5,11.10^{5}-\frac{a_{3} \tau^{2}}{10,22.10^{5}}$ & $\frac{a_{3} \tau^{2}}{10,22.10^{5}}$ \\
\hline$(0,2,0,1)$ & $-a_{4}$ & $-5,11.10^{5}+\frac{a_{4} \tau^{2}}{10,22.10^{5}}$ & $5,11.10^{5}-\frac{a_{4} \tau^{2}}{10,22.10^{5}}$ & $\frac{a_{4} \tau^{2}}{10,22.10^{5}}$ \\
\hline
\end{tabular}

Table I. Energy levels due to DNC space, where we suffice with eigenvalues corrections to the ground state, i.e. $E_{1,2}= \pm m c^{2}$.

\section{CONCLUSION}

All numerical values of the energy are in units of eV. It may be worth to underline that thanks to the Kronecker's delta, the elements of the perturbed Hamiltonian merely will take many values.

Now, the DNC and non-DNC effects on the energy levels of the DO are illustrated in fig. 3 .

The upper bound on the value of the NC parameter $\Theta$ is $\sqrt{\Theta} \leq 2 \times 10^{-20} \mathrm{~m}$ [39], as well for $\tau$ is $\sqrt{\tau} \leq 10^{-17} \mathrm{eV}$ [40]. The bound on $\sqrt{\tau}$ is consistent with the accuracy in the energy measurement $10^{-12} \mathrm{eV}$.

It is important to clarify that presence of $\tau^{2}$ in the eigenvalues is not due to the act of the second-order correction, but rather to the Dirac matrices in the perturbed Hamiltonian term.

In fig. 3, we see how the energy levels are splitting as the $\Theta$ and $\tau$ parameters turn on. Values of the eigenvalues $E_{1}$ and $E_{2}$ are shown in the table 1 . Firstly, in fig. 3 , we show the effect of NC space when the perturbation parameter is off $(\tau=0)$, where the effect of noncommutativity is very significative as explained in fig. 1 . Thereafter, the effect of noncommutativity is more significative when the DNC perturbation term is present $(\tau \neq 0)$, the presence of this term exhibits the energy shifts. The last part of fig. 3 shows the combined effect of both DNC and $\mathrm{NC}$ parameters, where the effect is very evident.
In conclusion, the DO has been investigated in twodimensions in presence of an external magnetic field in DNC space in terms of second quantization and through properly chosen canonical pairs of coordinates and its corresponding momenta in a complex NC space. However, the dynamical noncommutativity was treated as a perturbation. More precisely, we have solved the DO problem in two-dimensional $\mathrm{NC}$ space to find the exact energy spectrum and wave functions. Therefore, we have employed these obtained results to find the first-order correction to the eigenvalues and eigenvectors. So that the correction due to dynamical noncommutativity on the energy of the quantum system can be stated in terms of $\tau$. It is worth noting that we addressed the system in NC space as a fundamental system instead of considering the fundamental system in a commutative space and noncommutativity is a perturbation. The first-order correction for the ground state of the DO due to noncommutativity of space is zero for a non-DNC case while it has a nonvanishing value in DNC case. Knowing that the result reduces to that of usual DO in commutative space in the limits of $\tau \rightarrow 0, \Theta \rightarrow 0$.

As mentioned in section II, some operators in DNC space are non-Hermitian. This mixture of DNCS and the non-Hermiticity theory together with the string theory can lead to fundamental new insights in these three fields. Distinctly, there are plenty of interesting problems arising from our investigation, such as the investigation of further possibilities of consistent deformations, the construction of the solution for the DKP oscillator, as well Klein-Gordon oscillator. The study of additional models in terms this newly used DNC variables.
[1] Seiberg, N; Witten, E. String theory and noncommutativegeometry. J. High Energ. Phys. 1999, 9. https: //doi.org/10.1088/1126-6708/1999/09/032

[2] Gingrich, D.M. Noncommutative geometry inspired blackholes in higher dimensions at the LHC. J. High Energ. Phys.2010, 2010, 22. https ://doi .org/10.1007/ JHEP05 (2010) 022
[3] Nicolini, P. Noncommutative black holes, the final appeal to quantum gravity: a review. Int. J. Mod. Phys A 2009, 24(07), 1229-1308. https://doi.org/10.1142/ S0217751X09043353

[4] Gracia-Bondia. J. M. Notes on quantum gravity and noncommutative geometry: New Paths Towards Quantum Gravity.Springer, Berlin, Heidelberg, 2010. 3-58. 
https://doi.org/10.1007/978-3-642-11897-5_1

[5] Chaichian. M, Sheikh-Jabbari M. M, Tureanu. A. Hydrogen Atom Spectrum and the Lamb Shift in Noncommutative QED . Phys. Rev. Lett. 86(2001) 2716. https://doi.org/10.1103/PhysRevLett.86.2716

[6] Haouam, I. Analytical solution of $(2+1)$ dimensional Dirac equation in time-dependent noncommutative phase-space. Acta. polytech. 2020, 60(2), 111-121. https://doi.org/10.14311/AP. 2020.60.0111

[7] Haouam, I. On the noncommutative geometry in quantum mechanics. J. Phys. Stud. 2020, 24(2), 2002.https: //doi.org/10.30970/jps.24.2002

[8] Douglas. M.R, Nekrasov. N. A. Noncommutative field theory. Rev. Mod. Phys. 73 (2001) 977. https://doi. org/10.1103/RevModPhys .73.977

[9] Haouam, I. On the Fisk-Tait equation for spin-3/2 fermions interacting with an external magnetic field in noncommutativespace-time. J. Phys. Stud. 2020, 24, 1801. https://doi.org/10.30970/jps . 24.1801

[10] Snyder. H. S. Quantized space-time. Phys. Rev 71 :38 41, 1947. https://doi.org/10.1103/PhysRev.71.38

[11] Snyder. H. S. The electromagnetic field in quantized space-time. Phys. Rev $72: 68$ - 71, 1947. https://doi. org/10.1103/PhysRev.72.68

[12] Sasakura N. Space-time uncertainty relation and Lorentz invariance. JHEP 052000 (2000)015. https://doi.org/ 10.1088/1126-6708/2000/05/015

[13] Lukierski J, Ruegg H, Nowicki A and Tolstoi V N. Q deformation of Poincare algebra. Phys. Lett. B 264 (1991 ) 331-338. https://doi.org/10.1016/0370-2693(91) 90358-W

[14] Balachandran, A. P., \& Vaidya, S. (2007). Lectures on fuzzy and fuzzy SUSY physics. World Scientific. IISc $/ \mathrm{CHEP} / 11 / 05$

[15] Alavi, S.A., Rezaei, N. Dirac equation, hydrogen atom spectrum and the Lamb shift in dynamical noncommutative spaces. Pramana. J Phys 88, 77 (2017). https://doi .org/10.1007/s12043-017-1381-4

[16] Andreas Fring et al (2010). Strings from positiondependent noncommutativity. J. Phys. A: Math. Theor. 43 345401. https://doi.org/10.1088/1751-8113/43/ $34 / 345401$

[17] Gomes M and Kupriyanov V G 2009 Positiondependent noncommutativity in quantum mechanics. Phys. Rev. D79125011. https://doi.org/10.1103/ PhysRevD.79.125011

[18] Itô, D., Mori, K. \& Carriere, E. An example of dynamical systems with linear trajectory. Nuovo Cimento A (19651970) 51, 1119-1121 (1967). https ://doi .org/10.1007/ BF02721775

[19] Moshinsky. M, Szczepaniak. A, J. Phys. A: Math. Gen. 22, L817 (1989). https : //doi .org/10.1088/0305-4470/ $22 / 17 / 002$

[20] Martínez-y-Romero. R. P, Salas-Brito. A. L. Conformal invariance in a Dirac oscillator. J. Math. Phys. 33, 1831 (1992)https://doi.org/10.1063/1.529660

[21] Benitez. J, Martinez-y-Romero. P. R, Núnez-Yépez. H. N, Salas-Brito. A. L, Phys. Rev. Lett. 64, 1643 (1990). https://doi.org/10.1103/PhysRevLett.64.1643

[22] Quesne, C, Moshinsky, M. Symmetry Lie algebra of the
Dirac oscillator. J. Phys. A, Math. Gen.23, 2263 (1990). https : //doi .org/10.1088/0305-4470/23/12/011

[23] De Lange, O.L. Shift operators for a Dirac oscillator. J. Math. Phys.32, 1296 (1991). https ://doi .org/10.1063/ 1.529328

[24] Bermudez, A., Martin-Delgado, M.A., Solano, E. Phys. Rev. A76, 041801(R) (2007). https://doi.org/10. 1103/PhysRevA. 76.041801

[25] Bermudez, A., Martin-Delgado, M.A., Luis, A. Phys. Rev. A77, 063815 (2008). https://doi.org/10.1103/ PhysRevA.77.063815

[26] Franco-Villafane. J. A et al., First Experimental Realization of the Dirac Oscillator. Phys. Rev. Lett. 111, 170405 (2013). https://doi.org/10.1103/ PhysRevLett.111.170405

[27] Quimbay. C, Strange. P, arXiv:1311.2021.

[28] Quimbay. C, Strange. P, arXiv:1312.5251.

[29] Boumali. A. Thermodynamic properties of the graphene in a magnetic field via the two-dimensional Dirac oscillator. Phys. Scr. 90, 045702 (2015). https://doi.org/10. 1088/0031-8949/90/4/045702

[30] Bagchi. B and Fring. A 2009. Minimal length in quantum mechanics and non-Hermitian Hamiltonian systems. Phys. Lett. A3734307-10. https://doi.org/10.1016/j. physleta.2009.09.054

[31] Haouam, I. The Non-Relativistic Limit of the DKP Equation in Non-Commutative Phase-Space. Symmetry, 2019, 11, 223. https://doi.org/10.3390/sym11020223

[32] Mandal. B.P, Verma. S. Dirac oscillator in an external magnetic field. Phys. Lett. A 374 (2010) 1021. https: //doi.org/10.1016/j.physleta.2009.12.048

[33] Boumali. A., Hassanabadi. H. The thermal properties of a two-dimensional Dirac oscillator under an external magnetic field. Eur. Phys. J. Plus 128, 124 (2013). https : //doi .org/10.1140/epjp/i2013-13124-y

[34] Bermudez, A., Martin-Delgado, M.A., Solano, E. Phys. Rev. Lett.99, 123602 (2007). https://doi.org/10. 1103/PhysRevLett.99.123602

[35] Sakurai, J. J. (1994). Modern Quantum Mechanics (revised edition) Addison-Wesley.

[36] Cai, S., Jing, T., Guo, G. et al. Dirac Oscillator in Noncommutative Phase Space. Int $\mathrm{J}$ Theor Phys 49, 1699-1705 (2010). https://doi.org/10.1007/ s10773-010-0349-7

[37] Mandal, B. P, Rai, S. K. Noncommutative Dirac oscillator in an external magnetic field. Phys. Lett A, 376(36), 2467-2470 (2012). https://doi.org/10.1016/ j.physleta.2012.07.001

[38] Hassanabadi, H, Hosseini, S. S, Zarrinkamar, S. Dirac oscillator in noncommutative space. Chin Phys C, 38(6), 063104 (2014). https://doi.org/10.1088/1674-1137/ $38 / 6 / 063104$

[39] Bertolami, O., Rosa, J. G., De Aragao, C. M. L., Castorina, P., \& Zappala, D. Noncommutative gravitational quantumwell. Phys. Rev. D 2005, 72, 025010. https: //doi.org/10.1103/PhysRevD.72.025010

[40] Alavi, S.A., Nasab, M.A. Gravitational radiation in dynamical noncommutative spaces. Gen Relativ Gravit 49, 5 (2017). https://doi.org/10.1007/ s10714-016-2167-6 


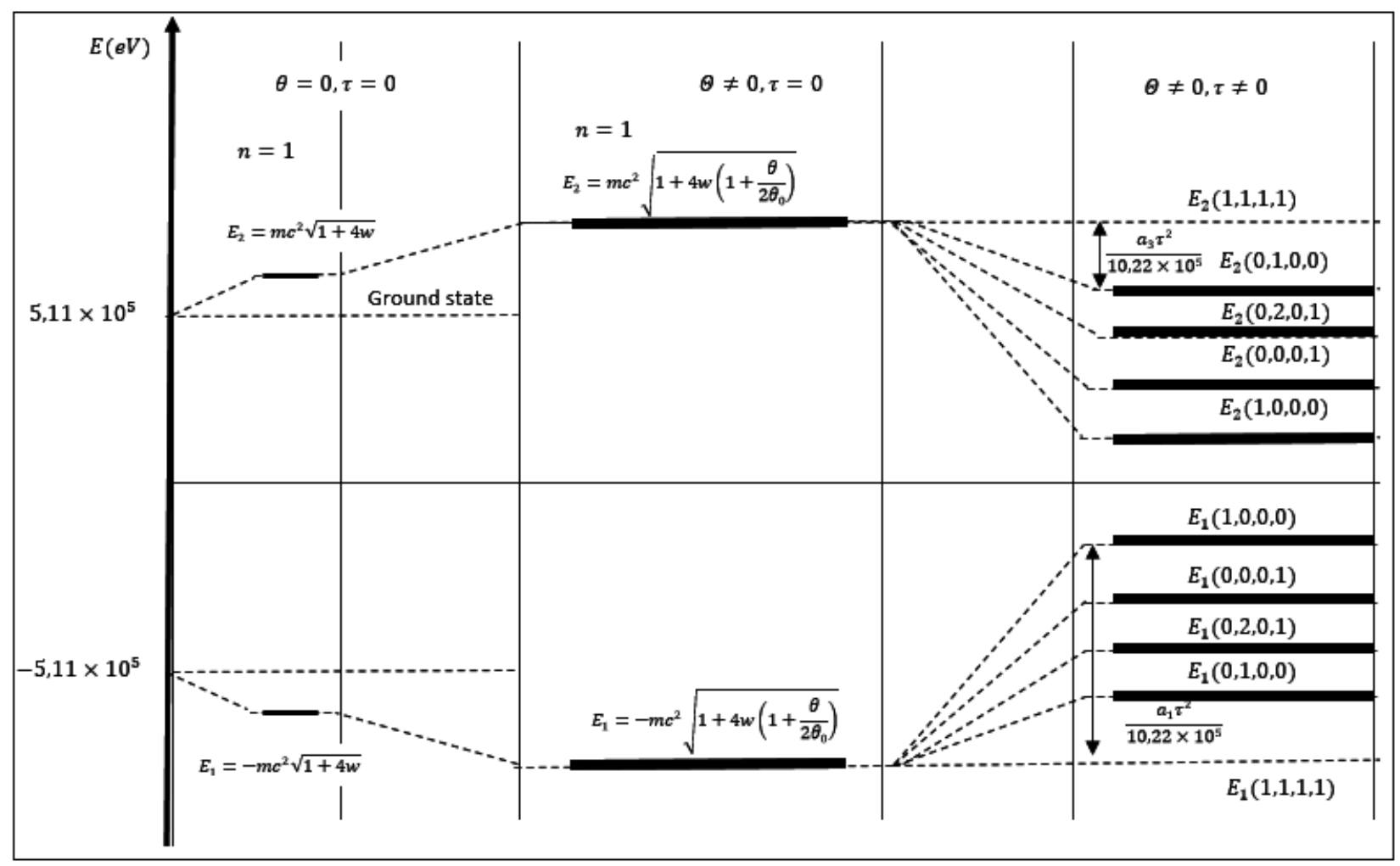

Figure 3. Diagram of splittings for energy levels due to DNC and non-DNC spaces. 